Supporting Information

\title{
Long Two-Dimensional Folding Chain Structure Formation of Poly(vinylidene fluoride) in Solutions of a Polar Solvent, $N$-methylpyrrolidone
}

\author{
Yuki Nohara1 ${ }^{1}$ Erika Saiki ${ }^{1}$, Toshiyuki Shikata ${ }^{1,2 *}$ \\ ${ }^{1}$ Cellulose Research Unit, Tokyo University of Agriculture and Technology, 3-5-8 Saiwai-cho, \\ Fuchu, Tokyo 183-8509, Japan \\ ${ }^{2}$ Division of Natural Resources and Eco-materials, Graduate School of Agriculture, Tokyo \\ University of Agriculture and Technology, 3-5-8 Saiwai-cho, Fuchu, Tokyo 183-8509, Japan
}

*To whom correspondence should be addressed.

e-mail: shikata@cc.tuat.ac.jp

Concise summaries of two theories used to calculate the magnitude of scattering vector $(q=\mid q)$ dependencies of particle form factors $(P(q))$ for randomly oriented rigid rods and rectangular columns in the main article.

Rigid Rod Particles ${ }^{\mathrm{SI} 1}$ : The main article cites as the reference 20. Particles included in this group are referred to "cylinders" in SasView ${ }^{\mathrm{S} 2}$ that is an open software used to calculate $P(q)$ in this study. The form factor, $P(q)$, for a rigid rod that has a length $(L)$ and a radius $(r)$ and is oriented randomly is theoretically calculated to be eq. (SI1). ${ }^{\mathrm{SI} 3, \mathrm{SI} 4}$ The parameter, $\alpha$, seen in eq. (SI1) represents an angle between the length axis and the scattering vector, $\boldsymbol{q}$.

$$
P(q)=\int_{0}^{\pi / 2}\left[\frac{\sin (q L \cos \alpha / 2)}{q L \cos \alpha / 2} \frac{2 J_{1}(q r \sin \alpha)}{q r \sin \alpha}\right]^{2} \sin \alpha \mathrm{d} \alpha
$$


where $J_{1}(x)$ means the first order Bessel function of a variable, $x$.

Rectangular Columns ${ }^{\mathrm{SI}}$ : The main article cites as the reference 18. Particles included in this kind are referred as "rectangular parallelepipeds" in the original article written by Nayuk and Huber ${ }^{\mathrm{SI}}$, and as simply "parallelepipeds" in SasView. According to Nayuk and Huber ${ }^{\mathrm{SI}}$ and Mittelbach and Porod ${ }^{\mathrm{SI}}$, the formfactor, $P(q)$, of a rectangular column that has a minor width, $W_{1}$, a major width, $W_{2}\left(>W_{1}\right)$, and a length, $L\left(>W_{2}\right)$ (cf. Figure SI1) and is randomly oriented can be theoretically calculated to be eq. (SI2). ${ }^{\text {SI4 }}$ The parameters, $\alpha$ and $\beta$, in eq. (SI2) represent angles between the length axis and the scattering vector, $\boldsymbol{q}$, and between the minor width direction and the $y$ axis of the detector plane, respectively.

$$
P(q)=\frac{2}{\pi} \int_{0}^{\pi / 2} \int_{0}^{\pi / 2}\left[\frac{\sin \left(q w_{1} \sin \alpha \cos \beta\right)}{q w_{1} \sin \alpha \cos \beta} \frac{\sin \left(q w_{2} \sin \alpha \cos \beta\right)}{q w_{2} \sin \alpha \sin \beta} \frac{\sin (q L \cos \alpha)}{q L \cos \alpha}\right]^{2} \sin \alpha \mathrm{d} \alpha \mathrm{d} \beta
$$

SasView can numerically calculate these form factors, $P(q)$, as a function of $q$ precisely.

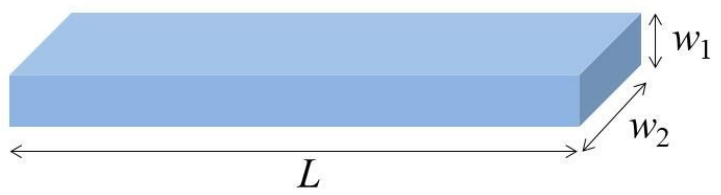

Figure SI1. Schematic depiction of a rectangular column with the minor width, $w_{1}$, the major width, $W_{2}$, and the length, $L$. 


\section{REFERENCES}

SI1) Fournet, G. Étude théorique et expérimentale de la diffusion des rayons X par les ensembles denses de particules. Bull. Soc. Fr. Mineral. Cristallogr. 1951, 74, 39-113.

SI2) SasView, https://www.sasview.org/

SI3) Guinier, A.; Fournet, G. Small-Angle Scattering of X-Rays. John Wiley and Sons: New York; 1955, Chapter 2.

SI4) Pedersen, J. S. Analysis of small-angle scattering data from colloids and polymer solutions: modeling and least-squares fitting. Adv. Colloid Interface Sci. 1997, 70, 171-210.

SI5) Nayuk, R.; Huber, K. Formfactors of Hollow and Massive Rectangular Parallelepipeds at Variable Degree of Anisometry. Z. Phys. Chem. 2012, 226, 837-854.

SI6) Mittelbach, P.; Porod, G. Zur Röntgenkleinwinkelstreuung verdünnter kolloider Systeme. Die Berechnung der Streukurven von Parallelepipeden. Acta Phys. Austriaca, 1961, 14, $185-211$. 